\title{
Anterior approach v. posterior approach - ultrasound-guided shoulder arthrogram injection
}

\author{
Merle Neethling-du Toit, Nat Dip Diag Rad, \\ Sports Radiol \\ Sports Science Institute of South Africa, Newlands, Cape Town
}

Richard de Villiers, MB ChB, DA, MMed Rad Drs Van Wageningen and Partners; Sports Science Institute of South Africa, Newlands, Cape Town

\begin{abstract}
Anterior approach ultrasound-guided arthrogram injections are a quick and reliable way of injecting contrast medium prior to a shoulder MRI scan. A retrospective study on patients who we had previously injected and scanned at our clinic was undertaken, to compare the more documented posterior ultrasound-guided approach with the anterior approach. The success rate for successful intra-articular injections for the anterior approach far exceeds the posterior approach. Additionally, the anterior approach is better tolerated by patients as the injections are much quicker and apparently less painful. In our experience, therefore, it is far better from every viewpoint to perform anterior approach ultrasound-guided shoulder arthrogram injections rather than posterior injections.
\end{abstract}

\section{Introduction}

The real-time ultrasound-guided anterior glenohumeral joint injection approach offers several advantages over the anterior fluoroscopic approach. The anterior fluoroscopic technique is commonly used to inject either a corticosteroid for management of frozen shoulder or contrast medium for computed tomography (CT) or magnetic resonance imaging (MRI) shoulder arthrography. ${ }^{1}$ Shoulder arthrography is a well proven technique, which can assist in the diagnosis of many diseases. ${ }^{2}$ If shoulder arthrography is performed in conjunction with MRI, detailed evaluation of the cartilaginous structures of the glenohumeral joint can also be undertaken. ${ }^{3}$

Fluoroscopically-guided arthrography is minimally invasive, but exposes patients to ionising radiation as well as the risk of needle misplacement. ${ }^{2}$ MRI arthrography preceded by fluoroscopically guided injection is time-consuming and impractical if the MRI scanner is too distant from the fluoroscopy room. ${ }^{4} \mathrm{~A}$ high-resolution ultrasound-guided technique for needle placement during shoulder arthrography using a posterior approach has been described by Cicak et al., and is commonly used by orthopaedic surgeons. ${ }^{5}$ Valls and Melloni evaluated the feasibility of MR arthrography of the shoulder with sonographic guidance using an anterior approach, which is the conventional radiological route. ${ }^{2}$

Our aim was to compare the anterior with the posterior ultrasoundguided arthrographic injection approach in achieving optimal needle placement, ensuring accuracy in shoulder arthrography injections.

\section{Materials and methods}

One hundred and thirty-five patients with clinical symptoms of shoulder instability presented for MRI arthrography of the shoulder, of whom 118 were injected using the anterior ultrasound-guided approach, and 17 were injected using the established posterior ultrasound-guided approach for pre-MRI contrast injection. A retrospective study was performed from existing departmental records. Patients gave written consent on the day of examination.

A Toshiba Nemio 20 ultrasound machine and a dedicated multifrequency linear transducer of $8-12 \mathrm{MHz}$ was used for all examinations.

\section{Anterior technique}

The patient lies supine, with the ultrasound machine on the same side as the shoulder being injected. The patient's arm is extended and the palm of the hand turned outwards or facing upwards, thus externally rotated. If this is too painful for the patient, the arm can be placed in a neutral rotation, with the palm against the thigh.

By externally rotating the arm, more anterior articular surface of the humeral head and increased intra-articular area for the needle insertion are exposed. It also ensures easier injection and that the long head of the biceps tendon is removed from the injection tract.

A transverse image of the anterior shoulder joint is obtained by using the coracoid process medially and the anteromedial humeral head laterally as reference points (Fig.1). The joint space should be clearly visualised in this plane. It is important to remember that the transducer must always be at a right angle to the proposed injection site and that

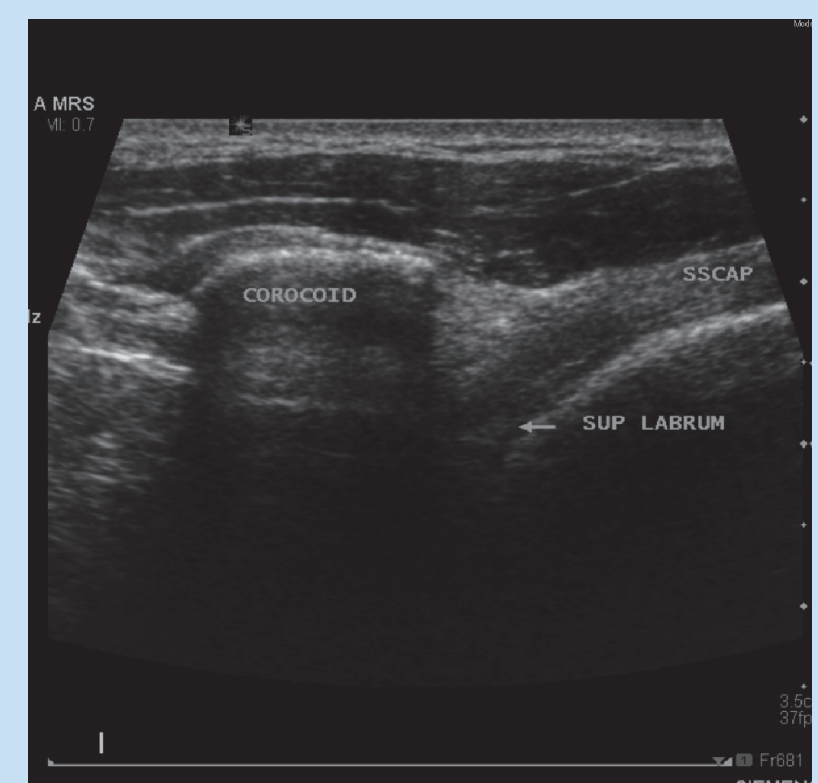

Fig. 1. Transverse ultrasound image of the shoulder. Note the coracoid process medially, the superior labrum and the subscapularis muscle and tendon, as labelled. 


\section{ORIGINAL ARTICLE}

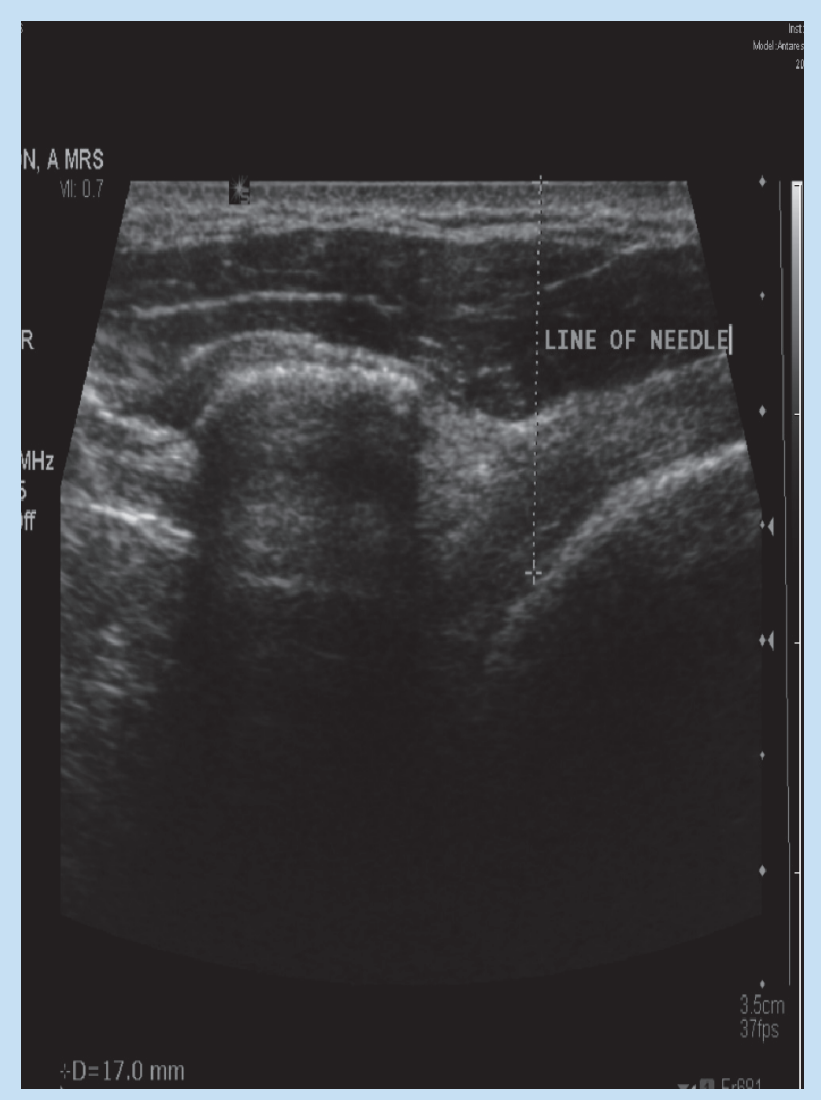

Fig. 2. Transverse ultrasound image of the shoulder. Note the intended direction of the needle placement through the rotator cuff interval.

the needle must not be angled superiorly or inferiorly, but rather kept perpendicular to the transducer to ensure accurate placement.

Using an aseptic technique and without moving the ultrasound transducer from the desired transverse plane, the needle is advanced into the joint space through the rotator cuff interval, using real-time ultrasound guidance (Fig.2). The needle is inserted into the shoulder approximately midway between the two reference points until it makes contact with the medial edge of the upper humeral head, with the bevelled edge of the needle facing towards the humeral head. A $4 \mathrm{~cm}$ green needle $(21 \mathrm{G})$ is used. For bigger shoulders, a yellow spinal needle can be used for extra length (19 G).

By using real-time ultrasound throughout this procedure, the intraarticular position of the needle tip and the subsequent complete distension of the shoulder joint can be visualised uninterruptedly. The needle tip can usually be clearly visualised and followed on the ultrasound monitor. Local anaesthetic is administered as a test-injection - low resistance while injecting confirms the intra-articular position of the needle. If high resistance is felt, the needle can be slightly angled medially away from the bony humeral surface. The administration of local anaesthetic can be watched carefully with the real-time ultrasound. Then, by simply changing syringes, the diluted gadolinium-saline contrast solution $(0.1$ $\mathrm{ml}$ gadolinium mixed with $20 \mathrm{ml}$ saline) is injected, while continuously watching on the real-time ultrasound monitor. Depending on the physical size of the shoulder joint, $15-20 \mathrm{ml}$ is injected. Retrograde flow from the needle after the syringe is disconnected indicates adequate joint distension.

\section{Posterior technique}

The patient lies semi-prone with the shoulder being injected elevated off the bed at approximately 45 degrees, with the arm superiorly and anteriorly opening the posterior shoulder joint. Some doctors prefer patients to sit with the affected arm stretched across the chest, with the hand of the affected shoulder resting on the opposite, unaffected shoulder.

Real-time ultrasound is similarly used to check the intra-articular needle position and shoulder joint distension after local anaesthetic and gadolinium administration. The ultrasound transducer is aligned in the long axis to the musculotendinous junction of the infraspinatus muscle, just inferior to the scapular spine, with the posterior glenoid rim and posterior glenohumeral joint line centred in the field of view. The transducer is angled and elevated to show the contours of the posterior glenoid rim, posterior glenoid labrum and humeral head. ${ }^{1}$ The same amounts of local anaesthetic and gadolinium-saline solution are administered as for the anterior technique described above.

\section{Results}

Needle placement using the anterior technique was accurate in $96 \%$ of applications, as determined by successful arthrography. Complete distension of the articular cavity was visualised on magnetic resonance images (MRIs) without damage to neighbouring structures. The needle placement and real-time ultrasound-guided contrast injection took an average of 5 minutes to complete for 3 different doctors.

Needle placement using the posterior technique was only accurate in $41 \%$ of applications, as determined by evaluating the post-injection MRI images. The average needle placement and real-time ultrasound-guided contrast injection took more than 5 minutes for 2 different doctors.

\section{Discussion}

Arthrographic MRI of the shoulder is known to increase diagnostic accuracy in comparison with conventional MRI. MRI arthrography is the most sensitive technique in the detection of shoulder joint abnormalities. ${ }^{6}$ However, it changes conventional MRI into a minimally invasive examination. By using real-time ultrasound, the procedure is not only quicker but, unlike the case of fluoroscopically-guided arthrography, there is no ionising radiation or administration of radio-opaque contrast medium involved. ${ }^{4}$

Various authors have described the posterior and/or anterior approach for needle placement, some using fluoroscopy and others ultrasound. The posterior approach is believed to be used more commonly by orthopaedic surgeons, while the anterior approach is the more conventional radiological procedure.

The technique of arthroscopy generally follows the posterior route. The path lies between the infraspinatus muscle and the teres minor muscle, with the point of entry $2 \mathrm{~cm}$ inferior and $1 \mathrm{~cm}$ medial to the 
posterior angle of the acromion. A potential risk is contact with the suprascapular nerve and the circumflex scapular vessels. If an entry point inferior to the teres minor muscle (through the quadrangular space) is used, other potential risks are contact with the axillary nerve and the posterior humeral circumflex artery. ${ }^{2,7}$

Anterior shoulder injections could result in penetration of the anterior stabilising structures of the glenohumeral joint. ${ }^{8}$ Using the anterior approach, which targets the rotator cuff interval, avoids the subscapularis muscle and tendon, inferior glenohumeral ligament and the antero-inferior labrum of the shoulder. This technique allows the needle to be inserted into the shoulder until it makes contact with the medial upper third of the humeral head, just lateral to the joint space, thus also avoiding the anterosuperior labrum. ${ }^{9}$ Using this technique also ensures that no contrast extravasation occurs, unless the amount of contrast injected exceeds the shoulder joint capacity. If the shoulder joint capsule ruptures, extravasation occurs in either the subscapularis recess or the biceps tendon sheath and not into the subacromial-subdeltoid bursa. ${ }^{10}$

Shumacher commented in 2003 that the standard anterior approach has performed surprisingly poorly in successful needle positioning and that many therefore prefer the posterior approach. He suggested that these approaches be compared in further studies and suggested that the posterior approach appeared preferable. ${ }^{11}$

In our experience, the anterior ultrasound-guided approach is quicker and easier to perform, more accurate, and better tolerated by patients than the posterior approach.

1. Zwar RB, Read JW, Noakes JB. Sonographically guided glenohumeral joint injection. Am J Radiol 2004; 183: 48-50.

2. Valls R, Melloni P. Sonographic guidance of needle position for MR arthrography of the shoulder. Am J Radiol 1997; 169: 845-847.

3. Jacobson JA, Lin J, Jamadar DA, Hayes CW. Aids to successful shoulder arthrography performed with a fluoroscopically guided anterior approach. Radiographics 2003; 23: 373-378.

4. Palmer WE. MR arthrography: is it worthwhile? Top Magn Reson Imaging 1996; 8: 24-43.

5. Cicak N, Matasovic T, Bajraktarevic T. Ultrasonographic guidance of needle placement for shoulder arthrography. J Ultrasound Med 1992; 11: 135-137.

6. Tirman PEJ, Applegate GR, Flannigan BD, et al. Magnetic resonance arthrography of the shoulder. Magn Reson Imaging Clin N Am 1993; 1: 125-142

7. Hulstyn MJ, Fadale PD. Arthroscopic anatomy of the shoulder. Othrop Clin N Am 1995; 26: 597-612.

8. Chung CB, Dwek JR, Feng S, et al. MR arthrography of the glenohumeral joint - a tailored approach. Am J Radiol 2001; 177: 217-219.

9. Dépelteau H, Bureau NJ, Cardinal E, et al. Arthrography of the shoulder: A simple fluoroscopically guided approach for targeting the rotator cuff interval. Am J Radiol 2004; 182: 329-332.

10. Bureau NJ, Cardinal E. Injection of rotator interval for shoulder arthrography - reply to letter. Am J Radiol 2004; 183: 1172-1173.

11. Schumacher HR. Aspiration and injection therapies for joints. Arthritis Rheum 2003; 49(3): 413-420.

\section{Radiology Positions Canada}

RADIOLOGIST: SK Radiology Associates of Regina is seeking Radiologists to join 16 member sole practice in Regina. RAR serves two acute care hospitals, its two clinics as well as numerous outside clinics and rural hospitals. Canadian Royal College certification or eligibility to write exam preferred. Locum positions are also available. Regina has a booming economy, no traffic and an international airport.

Contact Larry Kliman, Business Manager tel: 306-529-3305 or e-mail larry@rarsk.com 